\title{
The link between FOXJ1 expression level in bladder carcinoma and tumor recurrence
}

\author{
BING LIANG ${ }^{1}$, XINWEI HE ${ }^{2}$, DONGHAO SHANG ${ }^{3}$, YE TIAN $^{3}$ and ZONGWEN LIU ${ }^{4}$ \\ Departments of ${ }^{1}$ Oncology and ${ }^{2}$ Infection, The Second Affiliated Hospital of Zhengzhou University, Zhengzhou, \\ Henan 450052; ${ }^{3}$ Department of Urology, Beijing Friendship Hospital, Capital Medical University, Beijing 100050; \\ ${ }^{4}$ Department of Radiotherapy, The Second Affiliated Hospital of Zhengzhou University, Zhengzhou, Henan 450052, P.R. China
}

Received June 13, 2016; Accepted December 16, 2016

DOI: $10.3892 / \mathrm{ol} .2017 .7504$

\begin{abstract}
We studied the expression level of FOXJ1 in bladder epithelial carcinoma and its relation to clinical features and tumor recurrence. From January 2014 to June 2015, 66 patients with bladder epithelial carcinoma were enrolled in this study and their tumor and para-carcinoma tissue samples were collected. FOXJ1 positive expression rate was evaluated using immunohistochemical staining, and FOXJ1 mRNA expression level was measured by RT-PCR. RT-PCR and immunohistochemistry results showed that FOXJ1 expression level in tumor samples was significantly lower than that in para-carcinoma tissue samples. The median survival time in patients with positive expression of FOXJ1 was signifi cantly longer than that of patients with negative expression of FOXJ1. We also showed that FOXJ1 expression level was negatively correlated with neoplasm staging and tumor recurrence rate. We concluded that FOXJ1 was expressed in low quantities in bladder epithelial carcinoma, which was closely correlated with the biological characteristics of the tumor. FOXJ1 expression presents a promising application prospect for further exploration of the specific biological mechanism of FOXJ1 in regulating the occurrence and development of bladder epithelial carcinoma. FOXJ1 may be used as a new marker for early diagnosis and prediction of recurrence.

Introduction

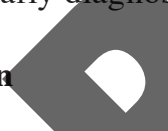

Bladder epithelial carcinoma is the most common malignant tumor of the urinary system without any certain pathogenesis. Usually, bladder epithelial earcinoma is multicenter and has infiltrative growth (1). Polyclonal theory argues that bladder epithelial carcinoma is a regional lesion of urothelium at

Correspondence to: Dr Zongwen Liu, Department of Radiotherapy, The Second Affiliated Hospital of Zhengzhou University, 2 Jing 8 Street, Zhengzhou, Henan 450052, P.R. China

E-mail: liuzongwenzdfy@126.com

Key words: bladder epithelial carcinoma, FOXJ1, median survival time, tumor recurrence different times and in different places (2). Presently, the molecular markers for early diagnosis of tumor and prediction of recurrence are insufficient. Results obtained from a prior study showed that FOX protein family is an important transcription factor family, having conventional DNA binding sites and extensively participating in various biological process such as differentiation, metabolism, apoptosis, proliferation, migration, invasion and the formation, development and regression of tumors (3). As a member of FOX family, FOXJ1 plays different roles in the occurrence and development of various tumors (4). FOXJ1 may play an inhibitory role in breast and ovarian eancers while its role in promoting the liver cancer has been reported (5). The present study further investigated the expression of FOXJ1 in bladder epithelial carcinoma and ts correlation with clinical features and tumor recurrence.

\section{Patients and methods}

Patient data. From January 2014 to June 2015, 66 patients with bladder epithelial carcinoma were enrolled in the present study. There were 39 males and 27 females with ages ranging from 42 to 67 years (average, $54.3 \pm 12.6$ years). In terms of TNM neoplasm staging, 27 cases were in early-stage, 25 cases in middle-stage and 14 cases were in late-stage. In terms of treatment methods, 40 cases underwent excision and chemotherapy and 26 cases had radiotherapy or chemotherapy. Patients' tumors and para-carcinoma tissues were collected (3 samples per patient collected by the same team), and tissue slices were prepared for observation. Patients with other primary malignant tumors and those who had difficulties with sample collection procedures were excluded. Study was approved by the Second Affiliated Hospital of Zhengzhou university Ethics Committee and written informed consent was obtained from the patients.

\section{Observation indexes and detection methods}

Observation of the positive expression rate of FOXJ1 by immunocytochemistry. SP immunohistochemistry was conducted using the following reagents and instruments: Anti-human FOXJ1 monoclonal antibody (Abcam, Cambridge, UK), Rat SP ELISA kit, DAB ELISA kit, hematoxylin and neutral balsam (Beijing Zhongshan Golden Bridge Biotechnology Co., Ltd., Beijing, China); sample injector (Eppendorf AG, Hamburg, 
Germany), slide warmers (Hubei Huida Instruments Co., Ltd., Xiaogan, Hubei, China), light microscope and BX-UCB digital slicing virtual scanner (Olympus Corp., Tokyo, Japan).

Main procedures were as follows: Samples dewaxing was done using xylene and hydrating using gradient ethanol. For antigen retrieval, samples were immersed in $0.01 \mathrm{~mol} / \mathrm{l}$ citrate buffer solution for 2 min under pressure at boiling temperature and then cooled down at room temperature. Samples were washed with PBS buffer solution (5 min of washing, 3 times) followed by incubation in $3 \% \mathrm{H}_{2} \mathrm{O}_{2}$ at room temperature for $20 \mathrm{~min}$. Samples were washed again in PBS buffer solution (5 min of washing, 3 times). To block nonspecific bindings, normal goat serum working solution was added drop by drop on the samples and samples were incubated at room temperature for $30 \mathrm{~min}$ in a humid environment. Primary antibody was added (1:400 anti-human FOXJ1 monoclonal antibody) followed by overnight incubation at $4^{\circ} \mathrm{C}$ in a humid environment. Primary antibody was replaced with normal mouse IgG in the negative control. Samples were washed with PBS buffer solution (5 min of washing, 3 times) followed by adding secondary antibody. Samples were then incubated for $20 \mathrm{~min}$ in a humid environment. Samples were then washed with PBS buffer solution (5 min of washing, 3 times). Streptavidin working solution with horseradish peroxidase was added, followed by DBA color-developing, counterstaining with hematoxylin, hydrochloric acid alcohol differentiation, coloring ammonia back to blue, hydrating gradient ethanol, hyalinizing xylene, neural balsam sealing and drying temperature.

Under the premise that the the clinicopat of the patients were unknown to our 2 pa immunohistochemical results were analyzed using Sinicrope grading standards. Semiquantitative method was used, and the comprehensive scores were judged using the intensity of the color and the proportion of stained cells (1).

Determination of staining intensity: Non-positive staining, 0 point, faint staining 1 point, moderate-intensity staning, 2 points, strong staining,

Determination of the proportion of positive cells: $\leq 5 \%$ positive cells, 0 point, $6-25 \%$ positive cells, 1 point, $26-50 \%$ positive cells, 2 points, $51-75 \%$ positive cells 3 points, $>75 \%$ positive cells, 4 points (3). Final grading in each sample according to staining intensity and the proportion of stained cells were scored: Negative ( $0-3)$ and positive (4-12).

RT-PCR quantitative detection of FOXJ1 mRNA expression level. Main reagents and instruments used were the following: TRIzol ${ }^{\circledast}$ Reagent (Invitrogen Life Technologies, Shanghai, China), DNA reverse transcription kit (Thermo Fisher Scientific, Inc., Beijing, China), SYBR-Green Master Mix (Takara Bio, Dalian, China), homogenizer (Thermo Fisher Scientific, Inc.), low-temperature high-speed centrifuge (Hunan Xiangya Instrument General Plant), NanoDrop ${ }^{\circledR}$ ND-1000 ultraviolet spectrophotometer (Thermo Fisher Scientific, Inc.), low-profile $0.2 \mathrm{ml} 8$-tube strips without caps and Optical Flat 8-cap strips, for $0.2 \mathrm{ml}$ tubes and plates (ultraclear) (Bio-Rad, Berkeley, CA, USA), real-time PCR instrument (Bio-Rad, Hercules, CA, USA).

Total RNA was extracted using Normal TRIzol method and RNA purity and concentration were verified with spectrophotometery. cDNA was synthetized using First Strand cDNA Synthesis kit. The following FOXJ1 primers were designed with primer 5: FOXJ1 forward, 5'-TGGATCACGGACAAC TTCTGCTA-3' and FOXJ1 reverse, 5'-CACTTGTTCAGA GACAGGTTGTGG-3'; GAPDH forward, 5'-GCACCGTCA AGGCTGAGAAC-3' and GAPDH reverse, 5'-TGGTGAAGA CGCCAGTGGA-3'. We used $10 \mu 1$ of X SYBR ${ }^{\circledR}$ Premix Ex Taq ${ }^{\mathrm{TM}}, 1 \mu 1$ of PCR positive and negative primers, respectively, $2 \mu \mathrm{l}$ of cDNA template and $6 \mu \mathrm{l}$ of $\mathrm{ddH}_{2} \mathrm{O}$ (total volume, $20 \mu \mathrm{l}$ ). Reaction condition was: $95^{\circ} \mathrm{C}$ for $30 \mathrm{sec}, 95^{\circ} \mathrm{C}$ for $5 \mathrm{sec}, 60^{\circ} \mathrm{C}$ for $30 \mathrm{sec}, 72^{\circ} \mathrm{C}$ for $15 \mathrm{sec}$ for 40 cycles. For solubility curve analysis we used $95^{\circ} \mathrm{C}$ for $60 \mathrm{sec}, 55^{\circ} \mathrm{C}$ for $60 \mathrm{sec}$, cooling at $37^{\circ} \mathrm{C}$ for $1 \mathrm{~h}$. We used $2^{-\Delta \Delta \mathrm{CT}}$, ${ }^{\Delta} \mathrm{Ct}=\mathrm{Ct}$ FOXJ1-Ct GAPDH and $\mathrm{Ct}$ value was the cycle threshold required when the intensity of fluorescence signal reached the predicted value

Statistical processing. SPSS 19.0 (SPSS, Inc., Chicago, IL,
USA) software used for statistical analysis. Quantitative data were expressed as mean \pm standard deviation, and the comparison between two groups was made by t-test. Comparison between multiple groups was done by single factor ANOVA, Qualitative data were expressed by percentage (\%) and the comparison between groups was made using $\chi^{2}$ test. Median survival time was tested by Kaplan-Meier method and relevant analysis was made by Pearson or Spearman test. $\mathrm{P}<0.05$ was considered to indicate a statistically significant difference.

\section{Results}

The positive expression rate of FOXJ1. FOXJ1 protein was mainly located in karyon of para-carcinoma. It was mainly located in brain tumor tissues (Fig. 1). There were 61 cases of positive expression in para-carcinoma samples $(92.4 \%)$ and 32 cases of positive expression in tumor samples (48.5\%). Positive expression rate in tumor samples was significantly lower, and the difference was statistically significant $\left(\chi^{2}=30,607, \mathrm{P}<0.001\right)$. Positive expression rate in early-stage, middle-stage and late-stage patients was 63.0,32.0 and 28.6\%, respectively. Spearman relevant analysis results revealed that FOXJ1 expression was negatively correlated with neoplasm staging $\left(\chi^{2}=6,756, \mathrm{P}=0.034\right)$.

The expression level of FOXJ1 mRNA. FOXJ1 mRNA expression level in tumor samples was significantly lower than that in the para-carcinoma tissue samples, and the difference was statistically significant $[(0.42 \pm 0.03)$ vs. $(0.89 \pm 0.05), \mathrm{t}=15,627$, $\mathrm{P}<0.001]$. FOXJ1 mRNA expression levels in early-stage, middle-stage and late-stage patients were $0.66 \pm 0.66,0.53 \pm 0.06$ and $0.35 \pm 0.04$, respectively. Difference between groups was statistically significant $(\mathrm{F}=16,421, \mathrm{P}<0.001)$.

Correlation between FOXJ1 expression and median survival time and recurrence rate. Follow-up period was $\sim 6-22$ months (follow-ups ended by January 2016), and the median time was 15 months. The median survival time for patients with positive expression of FOXJ1 was significantly longer than that of patients with negative expression of FOXJ1 (22 vs. 13 months), and the difference was statistically significant (log-rank test 

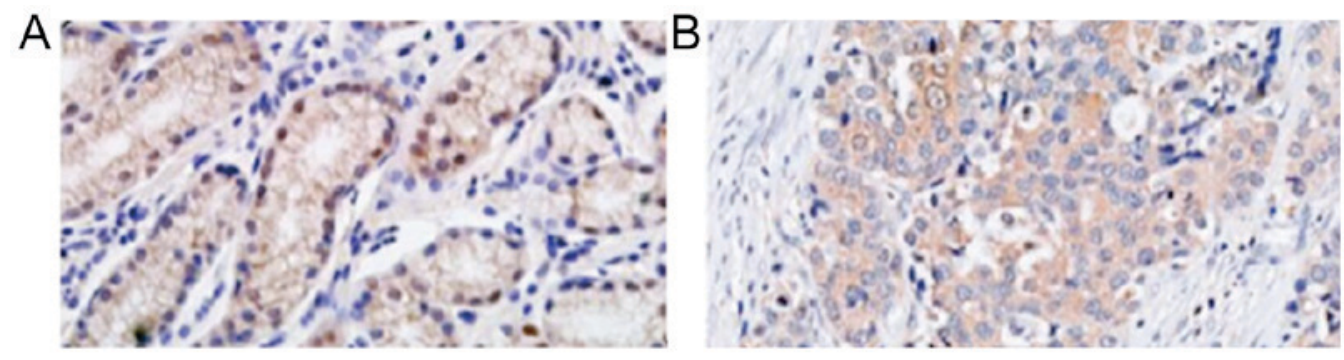

Figure 1. FOXJ1 expression in tumor and para-carcinoma tissues (x200), (A) para-carcinoma and (B) tumor.

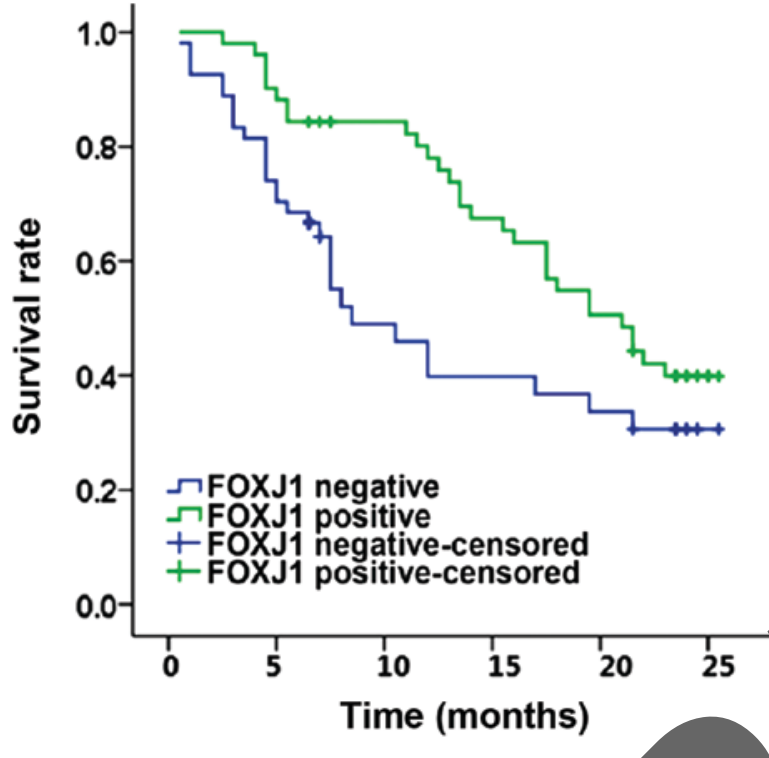

Figure 2. Analysis of FOXJ1 expression

regulation. FOX proteins can interact with multiple accessory factors involved in the regulation of cell differentiation, cell cycle progression and programmed cell death (9). FOX protein involvement in G1 stage retardation, G2 stage delay, DNA repair and apoptosis and inhibition of tumor cell proliferation has been reported $(10)$

FOX proteins seem to be participating in the biological regulation of various malignant tumors (11). It was shown that FOXM1 and FOXO3A compete for the same target sequence within promoter region of the same DNA and produce opposite transeriptional patterns (12). FOXJ1, also called HFH-4 or FKHL13, belongs to FOX transcriptional factor family, the gene is located at $17 \mathrm{q} 22-\mathrm{q} 25$, and the total length of its cDNA is $\sim 2.6 \mathrm{~kb}$. A recent study (13) reported that FOXJ1 was an important immunoregulatory transcriptional factor mainly expressed in $\mathrm{T}$ cells and its expression declined rapidly when $T$ cells were activated. It could inhibit the humoral immune response of B cell mediation. B cells with defective FOXJ1 gene could spontaneously form a germinal center and produce a pathogenic autoantibody to intensify immune response. Results obtained from a prior study showed (14) that silencing FOXJ1 expression was probably an important mechanism in breast cancer development. It has been shown that FOXJ1 can inhibit the transcriptional activity of $\mathrm{NF}-\kappa \mathrm{B}$ outside the human body. NF- $\kappa \mathrm{B}$ activation has been shown to be correlated with tumor formation, promoting the expression of anti-apoptotic proteins, upregulation of chemotactic factors and cellular adhesion molecules (15). NANOG is a transcriptional factor in stem cells and is involved in regulating ovarian cancer invasion and transference. FOXJ1 was shown to be the downstream regulating factor of NANOG (16). Multiple studies $(4,5)$ have reported abnormal expression of FOXJ1 in various tumors. It was shown that FOXJ1 expression level and function depended on the occurrence and the stages of the tumor. Therefore, the function of FOXJ1 in the occurrence, development and tumor invasion needs further research.

Results obtained in the present study revealed that the positive expression and the level of FOXJ1 mRNA expression in tumors were significantly lower than that in para-carcinoma tissues. The median survival time in patients with positive expression of FOXJ1 was significantly longer than that of patients with negative FOXJ1. Results obtained from relevant analysis showed that FOXJ1 expression was negatively correlated with neoplasm staging and tumor recurrence rate. FOXJ1 was expressed in low quantities in bladder epithelial carcinoma, which was closely correlated with the biological characteristics of the tumor. We believe that FOXJ1 plays an important role in regulating the occurrence and the development 
of bladder epithelial carcinoma. The above indicate that future studies may prove that FOXJ1 is appropriate to be used as the new marker for early detection as well as prediction of recurrence in the cases of bladder epithelial carcinoma.

\section{References}

1. Sha N, Xie L, Chen T, Xing C, Liu X, Zhang Y, Shen Z, Xu H, $\mathrm{Wu} \mathrm{Z}, \mathrm{Hu} \mathrm{H}$, et al: Impact of lymphovascular invasion on recurrence and progression rates in patients with pT1 urothelia carcinoma of bladder after transurethral resection. Onco Targets Ther 8: 3401-3406, 2015.

2. Schlegel JU, Pipkin GE, Nishimura R and Duke GA: Studies in the etiology and prevention of bladder carcinoma. J Urol 101: 317-324, 1969.

3. Caburet S, Vaiman D and Veitia RA: A genomic basis for the evolution of vertebrate transcription factors containing amino Acid runs. Genetics 167: 1813-1820, 2004.

4. Chen HW, Huang XD, Li HC, He S, Ni RZ, Chen CH, Peng C, Wu G, Wang GH, Wang YY, et al: Expression of FOXJ1 in hepatocellular carcinoma: Correlation with patients' prognosis and tumor cell proliferation. Mol Carcinog 52: 647-659, 2013.

5. Venugopalan SR, Amen MA, Wang J, Wong L, Cavender AC, D'Souza RN, Akerlund M, Brody SL, Hjalt TA and Amendt BA Novel expression and transcriptional regulation of FoxJ1 during oro-facial morphogenesis. Hum Mol Genet 17: 3643-3654, 2008.

6. Major ML, Lepe R and Costa RH: Forkhead box M1B transcriptional activity requires binding of Cdk-cyclin complexes for phosphorylation-dependent recruitment of p300/CBP coactivators. Mol Cell Biol 24: 2649-2661, 2004.

7. Ono M, Yaguchi H, Ohkura N, Kitabayashi I, Nagamura Y, Nomura T, Miyachi Y, Tsukada T and Sakaguchi S: Foxp3 controls regulatory T-cell function by interacting with AML1/Ranx1. Nature 446: 685-689, 2007.
8. Ma RY, Tong TH, Leung WY and Yao KM: Raf/MEK/MAPK signaling stimulates the nuclear translocation and transactivating activity of FOXM1. Methods Mol Biol 647: 113-123, 2010.

9. Postnikoff SD, Malo ME, Wong B and Harkness TA: The yeast forkhead transcription factors fkh1 and fkh2 regulate lifespan and stress response together with the anaphase-promoting complex. PLoS Genet 8: e1002583, 2012.

10. Laoukili J, Kooistra MR, Brás A, Kauw J, Kerkhoven RM, Morrison A, Clevers H and Medema RH: FoxM1 is required for execution of the mitotic programme and chromosome stability. Nat Cell Biol 7: 126-136, 2005.

11. Myatt SS and Lam EW: The emerging roles of forkhead box (Fox) proteins in cancer. Nat Rev Cancer 7: 847-859, 2007.

12. Karadedou CT, Gomes AR, Chen J, Petkovic M, Ho KK, Zwolinska AK, Feltes A, Wong SY, Chan KY, Cheung YN, et al: FOXO3a represses VEGF expression through FOXM1-dependent and -independent mechanisms in breast cancer. Oncogene 31: 1845-1858, 2012.

13. Lin L, Spoor MS, Gerth AJ, Brody SL and Peng SL: Modulation of Th1 activation and inflammation by the NF-kappaB repressor Foxj1. Science 303: 1017-1020, 2004.

14. Demircan B, Dyer LM, Gerace M,Lobenhofer EK, Robertson KD and Brown KD: Comparative epigenomics of human and mouse mammary tumors. Genes Chromosomes Cancer 48: 83-97, 2009.

15. Lu T and Stark GR: Cytokine overexpression and constitutive NFkappaB in cancer. Cell Cycle 3:1114-1117, 2004.

16. Siu MK, Wong ES, Kong DS, Chan HY, Jiang L, Wong OG, Lam EW, Chan KK, Ngan HY, Le XF, et al: Stem cell transcription factor NANOG controls cell migration and invasion via dysregulation of E-cadherin and FoxJ1 and contributes to adverse clinical outcome in ovarian cancers. Oncogene 32 : $3500-3509,2013$

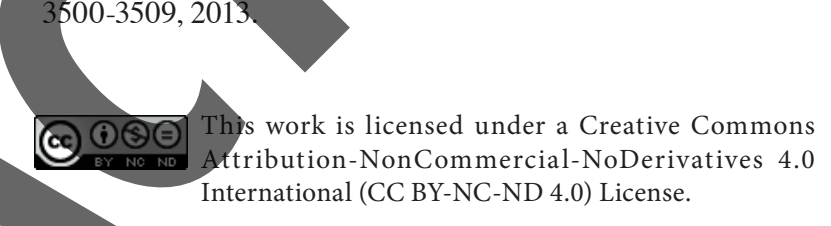

